\title{
OPEN Ketogenic Diet as a potential treatment for traumatic brain injury in mice
}

\author{
Meirav Har-Even ${ }^{1,2 \rrbracket}$, Vardit Rubovitch ${ }^{1}$, Whitney A. Ratliff ${ }^{3}$, Bar Richmond-Hacham ${ }^{1}$, \\ Bruce A. Citron ${ }^{4,5}$ \& Chaim G. Pick ${ }^{1,2,6,7}$
}

Traumatic brain injury (TBI) is a brain dysfunction without present treatment. Previous studies have shown that animals fed ketogenic diet (KD) perform better in learning tasks than those fed standard $\operatorname{diet}(S D)$ following brain injury. The goal of this study was to examine whether KD is a neuroprotective in TBI mouse model. We utilized a closed head injury model to induce TBI in mice, followed by up to 30 days of KD/SD. Elevated levels of ketone bodies were confirmed in the blood following KD. Cognitive and behavioral performance was assessed post injury and molecular and cellular changes were assessed within the temporal cortex and hippocampus. Y-maze and Novel Object Recognition tasks indicated that $\mathrm{mTBI}$ mice maintained on KD displayed better cognitive abilities than $\mathrm{mTBI}$ mice maintained on SD. Mice maintained on SD post-injury demonstrated SIRT1 reduction when compared with uninjured and KD groups. In addition, KD management attenuated mTBI-induced astrocyte reactivity in the dentate gyrus and decreased degeneration of neurons in the dentate gyrus and in the cortex. These results support accumulating evidence that KD may be an effective approach to increase the brain's resistance to damage and suggest a potential new therapeutic strategy for treating TBI.

Traumatic Brain Injury (TBI) is a brain dysfunction that occurs as the result of an external force's impact on the brain (external impact, penetration, or rapid head movement). The most common causes of TBI's are military injuries, road accidents, falls, assaults, and sports injuries ${ }^{1}$. Approximately 2.8 million people in the United States alone receive medical care for TBI each year, amounting to an annual cost above $\$ 76$ billion, including medical, unemployment, and additional costs ${ }^{1,2}$. Sequelae of TBI include physical, cognitive, behavioral, emotional, and social problems ${ }^{3,4}$. TBI emanates different pathophysiological changes that progress in two phases. The first, primary brain injury is a direct result of the external force applied to the brain; this phase consists of tissue alteration and damage proximal to the injury and interruption of axons and small vessels, which causes immediate necrotic neuronal cell death ${ }^{5}$. The secondary process initiated during the primary phase includes neuroinflammation, glutamate excitotoxicity and oxidative stress, which leads to neuronal apoptotic cell death ${ }^{5,6}$. Mild TBI (mTBI), which accounts for over $80-90 \%$ of all TBI cases, is difficult to diagnose because routine tests, including imaging, fail to show changes in brain structure ${ }^{7,8}$. Despite this, mTBI patients frequently suffer short and long-lasting cognitive, behavioral, and emotional impairments ${ }^{9}$. Such impairments include, among others, memory and concentration deficits, poor executive functions, depression, and anxiety-related disorders ${ }^{10}$.

The ketogenic diet (KD) is a high-fat, low carbohydrate diet, originally designed to stimulate the beneficial biochemical changes found in the fasting state, and has been used in patients with difficult-to-treat epilepsy since $1921^{11}$. KD is one method for inducing ketosis, a metabolic state in which the body uses ketone bodies as energy supply instead of glucose, the primary substrate for energy metabolism in the body and brain. Rodents receiving KD have shown a long-lasting affect of rapid increase in ketone body levels that lasted up to eight weeks into their $\operatorname{diet}^{12}$. Recent studies have begun to explore ketogenic therapies in other neurological and psychiatric disorders, including Parkinson's disease ${ }^{13}$ and Alzheimer's disease ${ }^{14,15}$. An increasing number of murine

\footnotetext{
${ }^{1}$ Department of Anatomy and Anthropology, Sackler Faculty of Medicine, Tel Aviv University, 6997801 Tel Aviv, Israel. ${ }^{2}$ Sylvan Adams Sports Institute, Tel Aviv University, 6997801 Tel Aviv, Israel. ${ }^{3}$ Laboratory of Molecular Biology, Bay Pines VA Healthcare System, Research and Development, 10000 Bay Pines Blvd., 151, Bldg. 22, Rm. 123, Bay Pines, FL 33744-4125, USA. 'Laboratory of Molecular Biology, Research and Development (Mailstop 15), VA New Jersey Health Care System, Building 16, Rm. 16-176, 385 Tremont Ave, East Orange, NJ 07018, USA. ${ }^{5}$ Department of Pharmacology, Physiology and Neuroscience, Rutgers-New Jersey Medical School, Newark, NJ 07103, USA. ${ }^{6}$ Sagol School of Neuroscience, Tel Aviv University, 6997801 Tel Aviv, Israel. ${ }^{7}$ Dr. Miriam and Sheldon G. Adelson Chair and Center for the Biology of Addictive Diseases, Tel Aviv University, 6997801 Tel Aviv, Israel. ${ }^{\boxplus}$ email: meiravhe@mail.tau.ac.il; pickc@tauex.tau.ac.il
} 

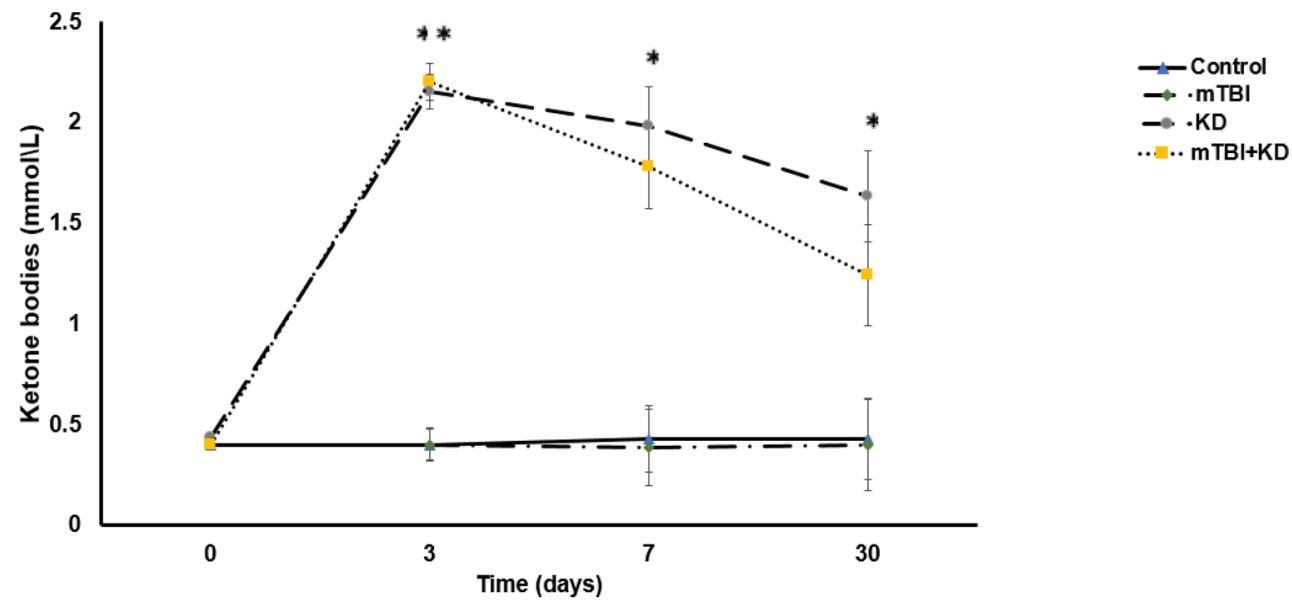

Figure 1. Levels of blood ketone bodies of control $(n=8)$, mTBI $(n=6), \operatorname{KD}(n=6)$, and mTBI $+\operatorname{KD}(n=5)$ mice. Mice that received KD (with or without $\mathrm{mTBI}$ ) demonstrated a prominent increase in ketone bodies compared to mice fed $\mathrm{SD}$ at 3,7 , and 30 days after the diet was initiated.

studies suggest that KD induces anti-inflammatory effects ${ }^{16}$. Studies have reported that $\mathrm{KD}$, and in particular, the ketone metabolite beta-hydroxybutyrate suppresses activation of the NLRP3 inflammation in response to several structurally unrelated NLRP 3 activators ${ }^{16}$ and improves the brain's ischemic tolerance ${ }^{17}$. KD management has also been shown to reduce activated microglial expression ${ }^{18}$. While mechanism of KD in neuroprotection is unknown, past reports found that KD increases glutathione level ${ }^{19}$ and Uncoupling protein (UCP) ${ }^{20}$ in cells after brain injuries, decreasing Reactive Oxygen Species (ROS).

A possible mechanism by which KD could be inducing neuroprotective effects is through the alteration of SIRT1 expression. SIRT1 has a role in developing the hippocampus by activating Akt and inhibiting GSK3 and is involved in various physiological processes such as oxidative stress response, genetic silencing, genome stability, and cell life extension ${ }^{21,22}$. The Sirtuin family of proteins is also active in the hypothalamus, where it plays a role in regulating circadian rhythm, endocrine pathways, and appetite ${ }^{23-26}$. Recently, several studies have shown that SIRT1 plays a significant role in induced neuroprotection following caloric restriction $(\mathrm{CR})^{27,28}$. CR has also been linked to reduced tau phosphorylation and maintenance of hippocampal neurons ${ }^{29}$, and related Tau pathology is known to be implicated in different types of $\mathrm{TBI}^{30,31}$. While $\mathrm{CR}$ has been shown to increase ketone bodies in the blood, KD does this at a higher level ${ }^{32}$. This is important to note as high fat diet and increased circulating ketone bodies have been shown to activate SIRT1 $1^{33}$. Thus, the effects of SIRT1 following KD, as in our model, may be more pronounced than in previous models utilizing CR. Our present study utilizing adult male ICR mice coincides with past rodent studies addressing SIRT $1^{34-36}$.

In the present study, we utilize a closed head weight drop model of murine mTBI to test the cognitive, cellular, and molecular effects of up to 30 days of KD management following injury. We report that KD initiated after mTBI ameliorated the cognitive deficits in spatial and visual memory, as well as cellular changes in neurons and glial cells induced by the injury. Our model also shows that KD sustained the levels of SIRT1 expression which were decreased with injury.

\section{Results}

KD increased the level of ketone bodies in blood. One-way repeated ANOVA revealed a significant main effect of time $\left[F(3,63)=28.31, p=0.000, \eta^{2}=0.57\right]$ and group $\left[F(3,21)=49.24, p=0.000, \eta^{2}=0.88\right]$ as well a time by group interaction $\left[F(9,63)=9.81, p=0.000, \eta^{2}=0.58\right]$. Consecutive simple effects analysis with Sidak test indicated that the level of ketone bodies in the blood of KD mice was significantly higher than the level in mTBI and control mice at the 3-day time-point (both $\mathrm{p}=0.000$ ), 7 -day time-point (both $\mathrm{p}=0.000$ ), and 30-day timepoint (both $\mathrm{p}=0.001$ ). Similarly, the blood ketone levels in the $\mathrm{mTBI}+\mathrm{KD}$ group were persistently higher than that of the mTBI and control groups at the 3 -day time-point (both $\mathrm{p}=0.000)$, 7-day time-point (both $\mathrm{p}=0.001$ ), and 30-day time-point $(p=0.022$ and $p=0.018$, respectively). See Fig. 1.

mTBI exposure and KD management does not affect anxiety. The EPM test was applied to assess anxiety-like behavior. Two-way ANOVA demonstrated no significant main effect of group $[\mathrm{F}(3,76)=1.61$, $\left.\mathrm{p}=0.194, \eta^{2}=0.06\right]$ or time-post injury $\left[\mathrm{F}(1,76)=3.33, \mathrm{p}=0.072, \eta^{2}=0.04\right]$, and no interaction between the two factors $\left[F(3,76)=0.55, p=0.649, \eta^{2}=0.02\right]$.

The mice's anxiety-like behavior was not affected either by the injury or diet management. See Fig. $2 \mathrm{~A}$.

KD ameliorates cognitive deficits. The NOR test was used to assess visual recognition memory. Twoway ANOVA revealed a significant main effect of group $\left[F(3,76)=17.11, p=0.000, \eta^{2}=0.40\right]$. Gabriel's post-hoc analysis demonstrated that the mTBI group performed significantly worse as compared to the controls, KD, 
A

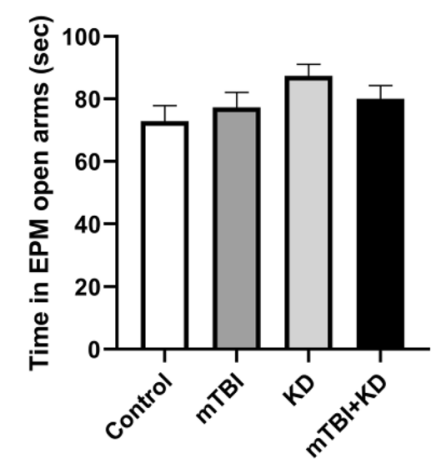

B

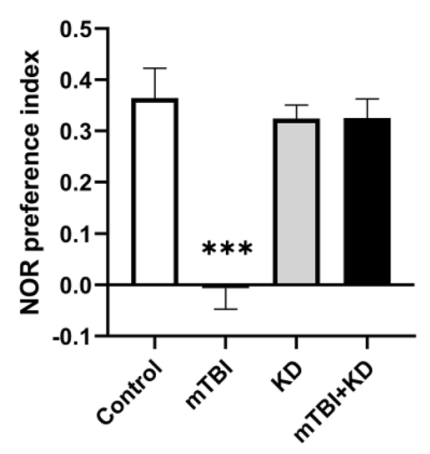

C

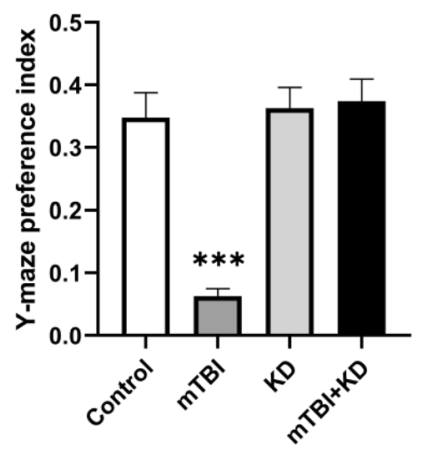

Figure 2. Behavioral tests scores. (A) EPM test-the time spent in open arms did not differ between mice in the control $(n=19), \operatorname{KD}(n=23)$, mTBI $(n=18)$, and mTBI + KD $(n=24)$ groups, indicating that mTBI did not affect anxiety-like behavior. (B) NOR test-differences in visual recognition memory performance between mice in the control $(n=19), K D(n=23)$, mTBI $(n=18)$, and mTBI + KD $(n=24)$ groups. $(C)$ Y-maze test- differences in spatial memory performance between mice in the control $(n=20), K D(n=24), \operatorname{mTBI}(n=19)$, and mTBI +KD $(n=26)$ groups.

and $\mathrm{mTBI}+\mathrm{KD}$ groups (all $\mathrm{p}=0.000$ ). There was no main effect of time-post injury $[\mathrm{F}(1,76)=0.60, \mathrm{p}=0.442$, $\left.\eta^{2}=0.01\right]$ or group by time-post injury interaction $\left[F(3,76)=2.30, p=0.084, \eta^{2}=0.08\right]$. See Fig. $2 B$.

The Y-maze test was used to assess spatial memory. Two-way ANOVA showed a significant main effect of group $\left[\mathrm{F}(3,81)=19.49, \mathrm{p}=0.000, \eta^{2}=0.42\right]$. Gabriel's post-hoc analysis showed that mice in the mTBI group performed significantly worse than mice in the control, KD, and $\mathrm{mTBI}+\mathrm{KD}$ groups $($ all $\mathrm{p}=0.000)$. There was no main effect of time-post injury $\left[F(1,81)=0.46 \mathrm{p}=0.498, \eta^{2}=0.01\right]$ nor a group by time-post injury interaction $\left[\mathrm{F}(3,81)=0.75, \mathrm{p}=0.526, \eta^{2}=0.03\right]$. See Fig. $2 \mathrm{C}$.

KD prevents the reduction of SIRT1 expression following $\mathrm{mTBI}$. SIRT1 expression within the hippocampus- one-way ANOVA revealed significant between-group differences in SIRT1 expression $[\mathrm{F}(3,21)=5.45$, $\left.\mathrm{p}=0.006, \eta^{2}=0.44\right]$. Gabriel's post-hoc analysis showed that the mTBI group had significantly lower SIRT1 levels than the control $(\mathrm{p}=0.009), \mathrm{KD}(\mathrm{p}=0.038)$ and $\mathrm{mTBI}+\mathrm{KD}(\mathrm{p}=0.025)$ groups. See Fig. $3 \mathrm{~A}$.

SIRT1 expression within the cortex-one-way ANOVA revealed significant between-group differences in SIRT1 expression $\left[\mathrm{F}(3,21)=6.26, \mathrm{p}=0.003, \eta^{2}=0.47\right]$. Gabriel's post-hoc analysis showed that the mTBI group had significantly lower SIRT1 levels than the mTBI +KD group $(\mathrm{p}=0.002)$. In addition, the mTBI group showed a marginally significant trend toward lower SIRT1 levels than the than the control $(p=0.090)$ and KD $(p=0.077)$ groups. See Fig. 3B.

Ketogenic Diet prevents mTBI-induced neuronal loss. The number of NeuN + neurons in the cortex and dentate gyrus-one-way ANOVA revealed significant between-group differences in the number of NeuN + neurons both within the cortex $\left[\mathrm{F}(3,16)=5.06, \mathrm{p}=0.012, \eta^{2}=0.49\right]$ and the dentate gyrus $[\mathrm{F}(3$, $\left.16)=8.51, p=0.001, \eta^{2}=0.61\right]$. Gabriel's post-hoc analysis demonstrated that the total number of NeuN + neurons within the cortex was significantly lower in the mTBI group than in the control $(\mathrm{p}=0.018)$ and $\mathrm{mTBI}+\mathrm{KD}$ groups $(\mathrm{p}=0.028)$. The total number of $\mathrm{NeuN}+$ neurons within the dentate gyrus was significantly lower in the mTBI group than in the control $(\mathrm{p}=0.008), \mathrm{KD}(\mathrm{p}=0.012)$, and $\mathrm{mTBI}+\mathrm{KD}(\mathrm{p}=0.002)$ groups. See Fig. $4(\mathrm{~A}-\mathrm{C})$.

Ketogenic Diet mitigates TBI-induced neuroinflammation by reducing reactive astrocytes. GFAP Intensity in the cortex and dentate gyrus- one-way ANOVA revealed significant between-group differences in GFAP intensity in the dentate gyrus $\left[F(3,16)=3.14, p=0.055, \eta^{2}=0.37\right]$, but not in the cortex [F $\left.(3,16)=2.60, p=088, \eta^{2}=0.33\right]$. Gabriel's post-hoc analysis demonstrated that the GFAP intensity in the dentate gyrus was higher in the mTBI group than in the mTBI+KD group $(\mathrm{p}=0.043)$. See Fig. 5A,B.

GFAP Morphology in the cortex and dentate gyrus-one-way ANOVA revealed significant between-group differences in GFAP Morphology in the dentate gyrus DGH- $\left[\mathrm{F}(3,16)=3.28, \mathrm{p}=0.048, \eta^{2}=0.38\right]$, but not in the cortex $\left[F(3,16)=0.52, p=0.674, \eta^{2}=0.09\right]$. Gabriel's post-hoc analysis revealed marked alterations in astrocyte morphology in the dentate gyrus of mTBI mice versus controls $(p=0.042)$. See Fig. 5(C,D).

Activated microglia expression in the cortex and dentate gyrus-one-way ANOVA revealed no significant differences between groups in the number of activated microglia within the cortex $[\mathrm{F}(3,16)=2.37, \mathrm{p}=0.108$, $\left.\eta^{2}=0.31\right]$ or dentate gyrus $\left[F(3,16)=2.15, p=0.134, \eta^{2}=0.29\right]$. See Fig. 5E,F.

Images of immunohistochemical staining in the DGH and the temporal cortex with GFAP positive cells shown in green, Iba-1 positive cells shown in yellow, and NeuN + cells in red. See Fig. 5G. 

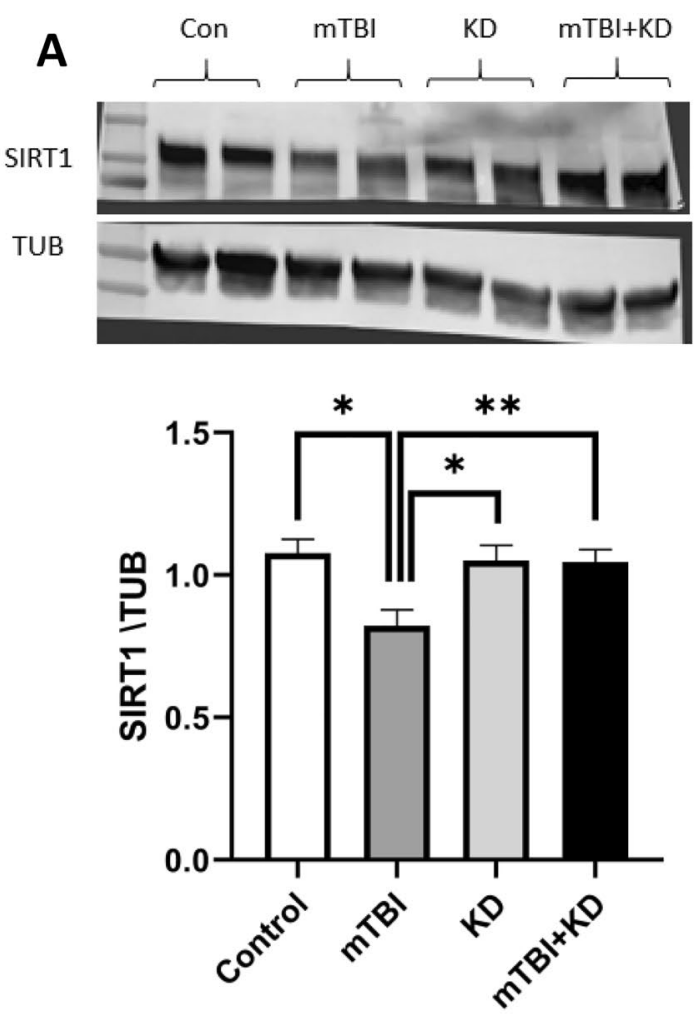

Hippocampus
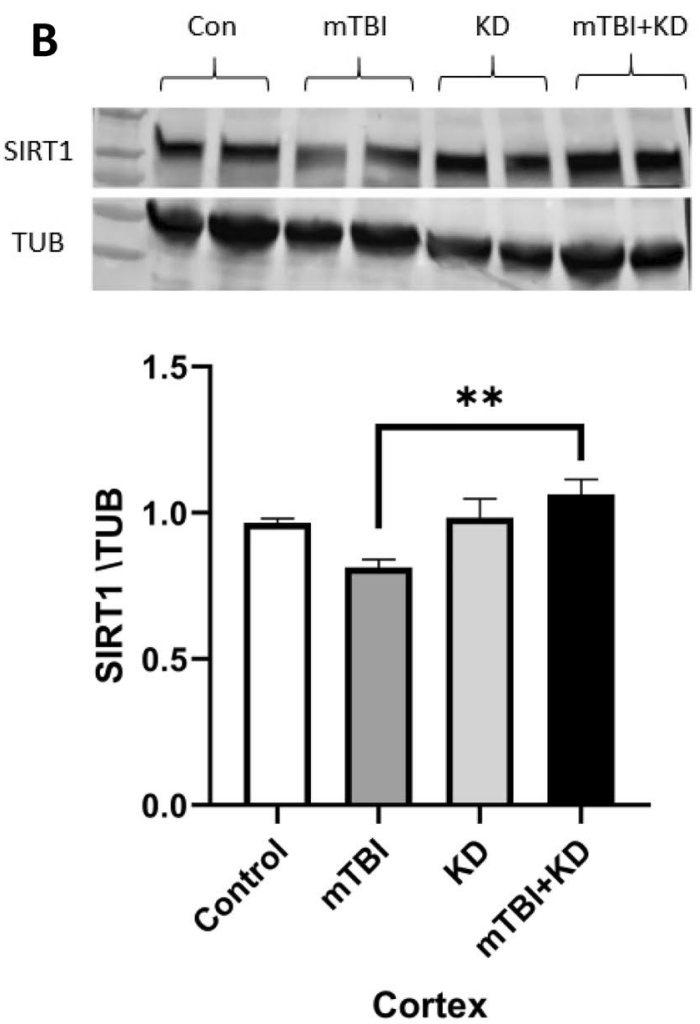

Figure 3. Changes in SIRT1 expression after mTBI in the cortex and hippocampus of control $(n=7), K D(n=5), \operatorname{mTBI}(n=6)$, and $\mathrm{mTBI}+\mathrm{KD}(\mathrm{n}=7)$ mice. (A) The levels of SIRT1 were significantly reduced in the hippocampus of mTBI mice compared with all groups. (B) The levels of SIRT1 were significantly reduced in the cortex of mTBI mice compared with all mTBI + KD group. The membrane was cropped immediately after the transfer stage due to the usage of two different antibodies on the same blot membrane, SIRT1 band size was at $110 \mathrm{kDa}$ and $\alpha$-tubulin band size was at $55 \mathrm{kDa}$, full-length blots/gels are presented in Supplementary Figs. 1-4. 
A

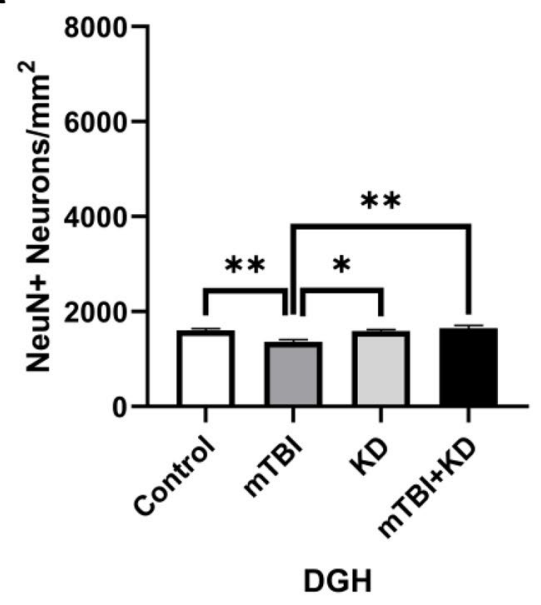

C

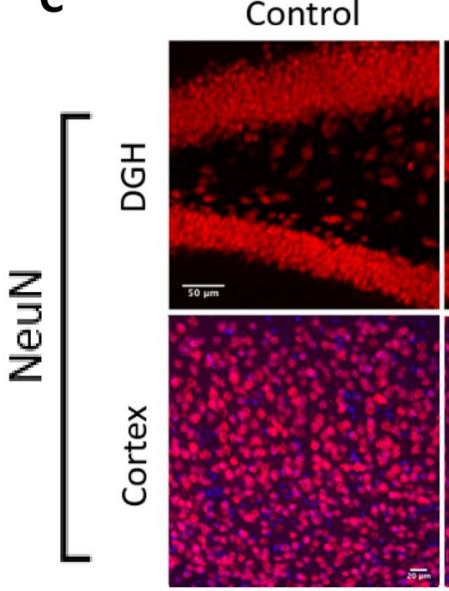

B

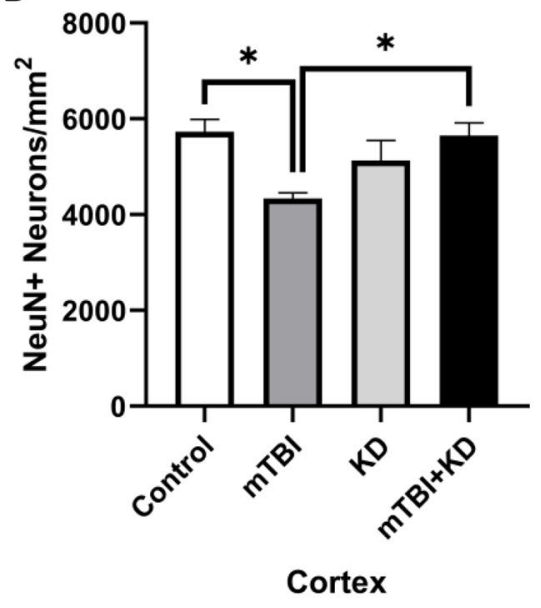

Figure 4. $\mathrm{NeuN}^{+}$changes in the cortex and hippocampus of control $(n=5), \mathrm{KD}(\mathrm{n}=5), \mathrm{mTBI}(\mathrm{n}=5)$, and $\mathrm{mTBI}+\mathrm{KD}(\mathrm{n}=5)$ mice. $(\mathbf{A}, \mathbf{B}) \mathrm{mTBI}$ induction led to a significant decrease in the density of $\mathrm{NeuN}^{+}$neurons compared to sham tissues in all regions tested. With $\mathrm{KD}$, there were significantly greater numbers of $\mathrm{NeuN}^{+}$ neurons than in the untreated mTBI mice, suggesting an increase in neuronal survival following the injury. (C) Representative images of immunohistochemical staining in the DGH and the temporal cortex are presented. NeuN positive cells are shown in red, and nuclei in blue; the scale bars are $50 \mu \mathrm{m}$ in the DGH and $20 \mu \mathrm{m}$ in the temporal cortex.

\section{Discussion}

TBI is a leading cause of death and long-term disability in the developed world, with more than 10 million people suffering worldwide every year ${ }^{37}$. The majority of these TBIs (80-95\%) are mild in nature ${ }^{7,8}$. TBI symptoms can occasionally resolve within the first year after injury, but up to $70-90 \%$ of patients continue to manifest prolonged and often permanent neurocognitive dysfunction. In light of the growing reports suggesting beneficial effects of $\mathrm{KD}$ in many neurological disorders ${ }^{13-15}$, the primary goal of this study was to assess the benefits of KD in mTBI. Our results suggest that KD may be a vital treatment modality, mitigating TBI-induced cognitive impairments, neuronal loss, and neuroinflammation in the closed-head mTBI mouse model.

We delivered a mild TBI to mice followed by up to 30 days of KD or SD. We were able to confirm that mice who received $\mathrm{KD}$ (with or without $\mathrm{mTBI}$ ) demonstrated a prominent increase in ketone bodies compared to mice fed SD at 3, 7, and 30 days after the diet. Previous studies have demonstrated that mice challenged with mTBI show cognitive impairments in visual and spatial memory ${ }^{10,38}$. Our previous published studies as well as the present study, ruled out the possible involvement of anxiety (as assessed by EPM) in the cognitive performance of the injured mice. This study's results replicated the previous findings regarding both visual and spatial memory while demonstrating that KD significantly ameliorated these cognitive deficits. These results support previous studies on the benefits of KD on brain injury in the short-term (7 days); rats who were fed KD exhibited evidence of neuroprotection after head trauma in the cortex and hippocampus ${ }^{39}$ and improvement in motor performance 
A

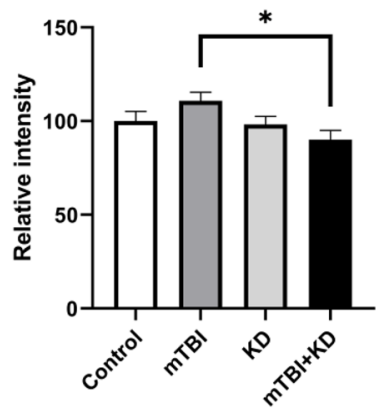

DGH

$\mathbf{E}$

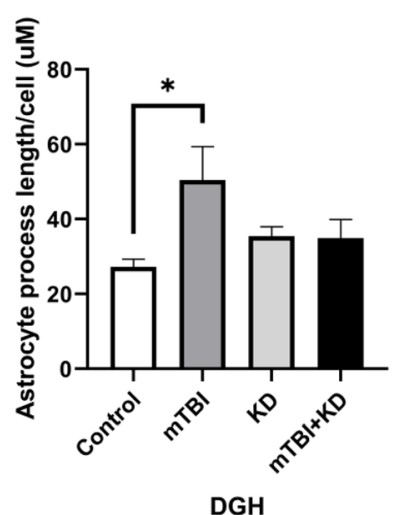

B

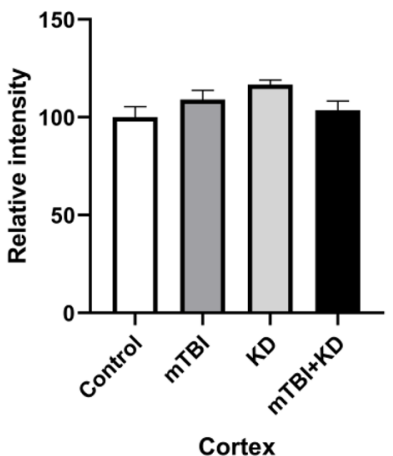

$\mathbf{F}$

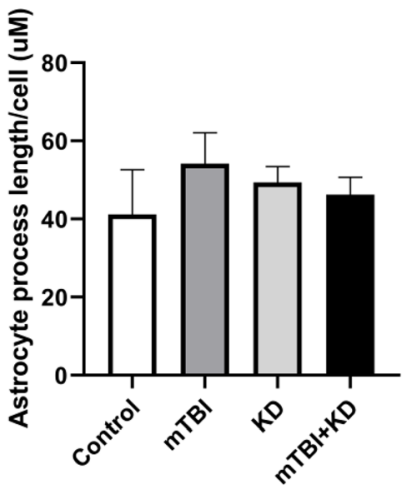

Cortex
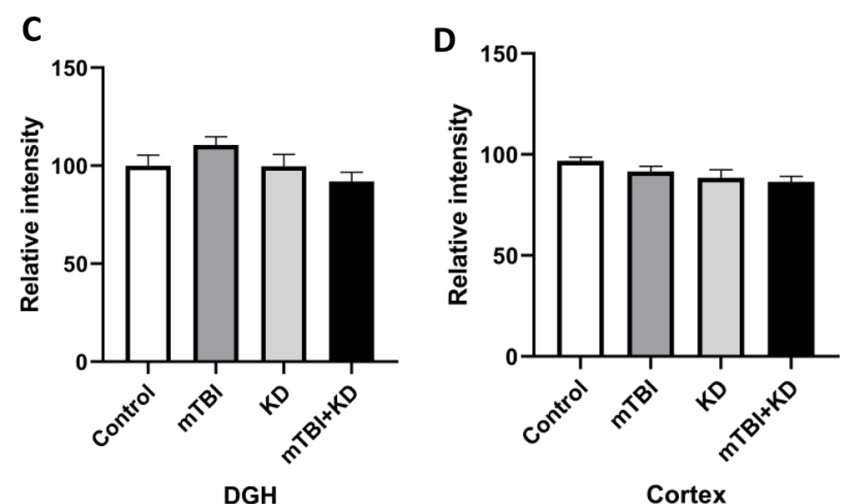

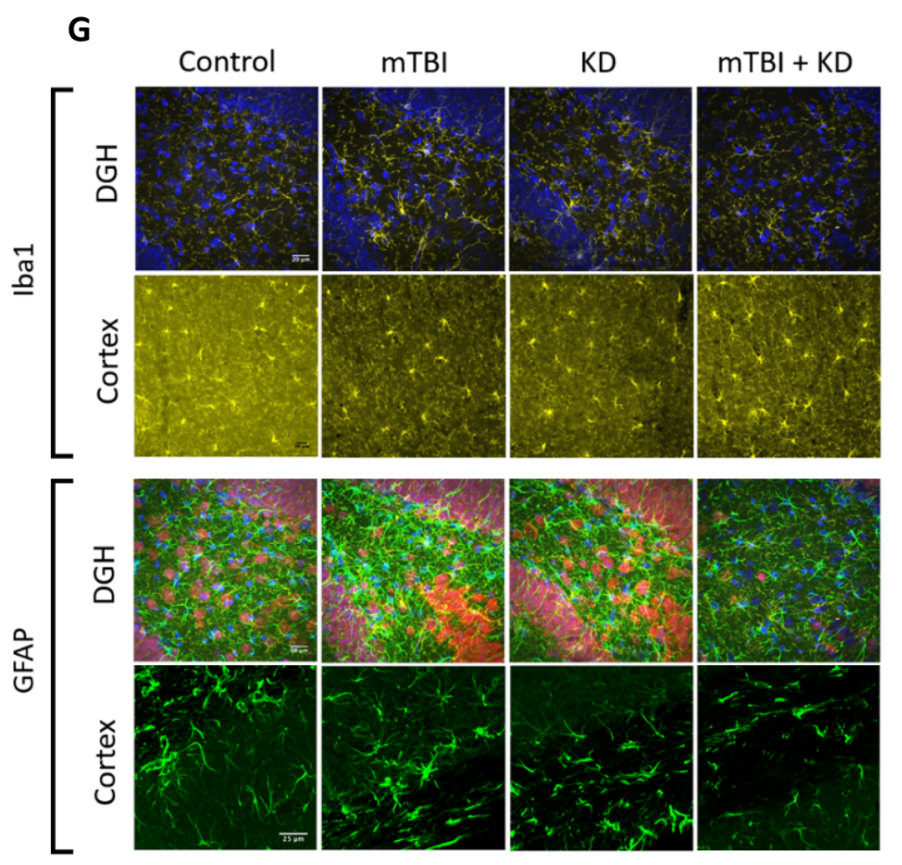

Figure 5. Microglia and astrocyte changes in the cortex and hippocampus of control $(n=5), K D(n=5)$, $\operatorname{mTBI}(n=5)$, and mTBI $+K D(n=5)$ mice. KD reduces the mTBI-induced elevation in active astrocyte expression in the DGH only and not in activated microglia expression at 30 days post-injury. Graphs present quantification of the total surface labeled with (A) GFAP in the DGH (B) GFAP in the temporal cortex (C) Iba-1 in the DGH (D) Iba-1 in the temporal cortex. In addition, (E,F) Astrocyte morphology in the DG Hilus region displayed approximately twice the ramification as the control samples. (G) Representative images of immunohistochemical staining in the DGH and the temporal cortex are presented. GFAP positive cells are shown in green, Iba-1 positive cells are shown in yellow, and NeuN+cells in red. The scale bars are 20-25 $\mu \mathrm{m}$. 


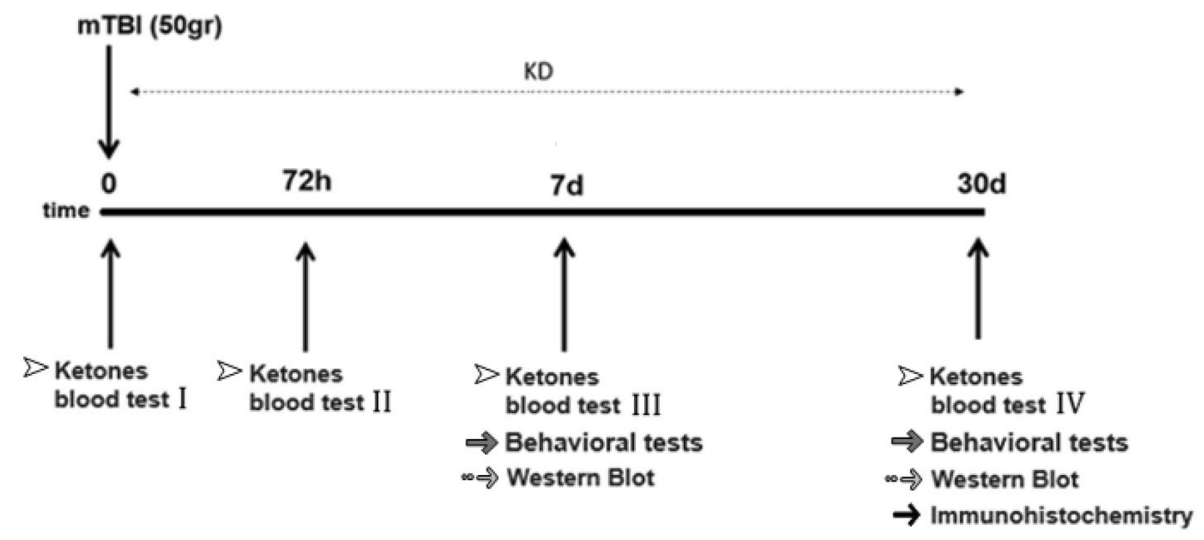

Figure 6. Study timeline. Animals were exposed to mTBI or sham and checked for ketone bodies in the blood at days $0,3,7$ and 30. Mice were then fed $\mathrm{KD} / \mathrm{SD}$ for 3, 7, or 30 days. Behavioral tests to assess behavior and cognitive abilities were carried out at 7 and 30 days following mTBI in separate cohorts. Western blot analysis to assess changes in SIRT1 levels following mTBI and diets management was performed at 7 and 30 days postmTBI. Immunohistochemical staining to evaluate neurodegeneration and neuroinflammation was performed at 30 days following the mTBI challenge.

in Beam balance and Beam walk tests ${ }^{18}$, $\mathrm{KD}$ administration has also been shown to reduce brain edema and cellular apoptosis $72 \mathrm{~h}$ after $\mathrm{TBI}^{40}$ and has demonstrated anti-tumor effects within the brain in rodent models ${ }^{41}$.

To better understand the molecular effects of TBI and KD, we evaluated SIRT1 levels in two brain regions, cortex and hippocampus, which play crucial roles in memory formation. TBI reduced the levels of SIRT1 in cortex and in the hippocampus 30 days post-injury which was ameliorated by $\mathrm{KD}$, suggesting a potential mechanism contributing to cognitive impairment and improvement in cognitive symptoms when given $\mathrm{KD}^{42}$. In contrast, at 7 days post-injury SIRT1 levels were not reduced in the cortex and hippocampus. This supports past findings showing an elevation in SIRT1 levels 1 day prior to ischemia that gradually decreased over a period of 7 days ${ }^{36}$. Our study's results align with prior studies of intermittent fasting and caloric restriction for 30 days post-injury ${ }^{28}$ and with research involving KD and SIRT1 in health-enhancing, aging, longevity, and neurodegeneration in animal models ${ }^{43,44}$.

We have previously reported that our mTBI model decreases the neuronal survival 3 weeks post injury ${ }^{45}$. Similarly, in the present study, we have shown a significant increase in neuronal cell death that persists 30 days following injury in both cortex and hippocampus. KD prevented this neuronal cell death. These results are consistent with an in vitro study that found the ketone body beta-hydroxybutyrate reduced axonal degeneration in diffuse axonal injury ${ }^{46}$. Additionally, we saw a marked increase in reactive astrocytes in hippocampus 30 days following injury, which was ameliorated by KD. We have previously reported that our mTBI model induces fundamental neuroinflammatory changes, including elevations in astrocyte reactivity, pro-inflammatory cytokine TNF- $\alpha$ levels, and expression of genes involved in inflammatory processes in several regions of the brain ${ }^{44,47-50}$. We can conclude that KD prevented the injury-induced neuro-inflammation, which is in agreement with previous studies that reported KD inhibited NLRP3 inflammasome activation, thus exerting neuroprotective effects ${ }^{17}$.

Our closed head injury mouse model of mild TBI was able to recapitulate the cognitive deficits observed in human TBI patients $\mathrm{s}^{51,52}$ and showed that ketogenic diet beginning following injury could protect against injury-induced memory loss. At the cellular level, we have demonstrated that mTBI induced neuronal cell death, astrocyte and microglial activation (neuro-inflammation), which were prevented in injured mice treated with KD. Our analysis of changes in SIRT1 suggests a viable molecular mechanism by which KD may be improving cognitive outcomes. Our results support accumulating evidence that KD may represent an important nonpharmaceutical treatment against the long-term molecular and cellular impacts of mTBI, which may ultimately improve cognitive symptoms and quality of life for TBI patients.

\section{Methods}

Experimental procedures. Mice were subjected to mTBI, and a ketogenic diet (KD) was initiated immediately for the following three timelines: 3 days, 7 days, and 30 days. Ketone bodies in the blood were measured $0,3,7$, and 30 days post-injury. Behavioral tests were performed at 7 or 30 days following mTBI in separate groups of animals. Western blot analysis was carried out on brain tissue collected from 7 and 30 days post mTBI. Immunohistochemical staining assessments were performed on brains that were collected 30 days post-injury. The timeline of the experimental procedures following exposure to mTBI is shown in Fig. 6.

Animals. The Sackler Commission on Animal Experimentation ethical committee approved the study and animal protocol 01-19-058 according to the Guidelines for Animal Experimentation of the National Institutes of Health (DHEW publication 23-85, revised, 1995). The study complies with ARRIVE guidelines.

Male ICR mice, aged 6-7 weeks, 30-40 g body weight, were acquired from Envigo RMS Israel. Mice were housed at 4-5 per home cage under a constant $12 \mathrm{~h} \mathrm{light/dark} \mathrm{cycle,} \mathrm{at} \mathrm{room} \mathrm{temperature}\left(22 \pm 2{ }^{\circ} \mathrm{C}\right)$ in a standard 


\begin{tabular}{|l|l|l|}
\hline \% Kcal from & Standard diet & Ketogenic diet \\
\hline Fat & 12.5 & 90.5 \\
\hline Carbohydrate & 77.4 & 0.3 \\
\hline Protein & 10.1 & 9.2 \\
\hline
\end{tabular}

Table 1. Macronutrient information of each diet.

plastic cage $\left(32 \times 21.5 \times 12 \mathrm{~cm}^{3}\right)$. Water and food was provided ad libitum, and the cages bedding, sterile sawdust, was replaced once per week. All mice were acclimatized to the facility for three days following transport and then moved into the experimental testing room for three days prior to experimentation. Animals were utilized only once throughout the study in either behavioral, biochemistry, or immunocytochemistry tests. Each animal in a given group was tested only once in order to avoid the possible confounding effect of behavioral testing. The number of animals evaluated in each assessment group and the measurement times performed was based on analysis of variance in our previous studies.

Mouse closed-head mild traumatic brain injury. Mild Traumatic Brain Injury (mTBI) was induced according to the closed-head weight-drop model, as employed in our previous studies ${ }^{53,54}$. The device consisted of an aluminum tube ( $80 \mathrm{~cm}$ in length and $13 \mathrm{~mm}$ in diameter). In the pre-injury stage, mice were anesthetized by inhalation of isoflurane and placed under the device on a sponge, the sponge supported the head of the mouse allowing some anterior-posterior motion, but no rotational head movement at the moment of impact. A metal weight $(50 \mathrm{~g})$ was dropped from the top of the tube to strike the head on the right temporal side between the ear and corner of the eye. Sham mice received anesthesia and were placed on the sponge for an equivalent length of time, but no weight was dropped. This model was chosen because it simulates traumatic head injuries such as road accidents or falls, as it imposes a diffuse and non-specific injury. Our model, while subject to a certain amount of variability due to the natural anatomic variation, still maintains a high degree of TBI injury similarity among all mice as seen in previous neuronal loss models ${ }^{53-56}$.

Ketogenic Diet. The macronutrient composition of the Ketogenic Diet (TD.96355; Harlan Teklad Laboratories, Madison, WI) was $9.2 \%$ protein, $0.3 \%$ carbohydrate, and $90.5 \%$ fat (\% kcal) (Table 1$)^{57-59}$. The corresponding components of the standard diet (SD) (TD.00606; Harlan Teklad Laboratories, Madison, WI) were $10.1 \%$ protein, $77.4 \%$ carbohydrate, and $12.5 \%$ fat $(\% \mathrm{kcal})^{57}$. The diets and water were provided ad libitum for 3,7 , and 30 days after the injury. Both diets were stored at $4{ }^{\circ} \mathrm{C}, \mathrm{KD}$, which had a solid, butter-like texture, was changed every day, and SD pellets were changed twice per week.

Measurement of Ketone Bodies. Ketone bodies in the blood were measured using the Precision Xtra Blood Glucose and Ketone Monitoring System (Abbott, Columbus, $\mathrm{OH}{ }^{60}$. Mice were anesthetized with isoflurane and tails were cut approximately $0.2 \mathrm{~mm}$ from the end, a drop of blood was squeezed directly into a testing strip attached to the measuring instrument. Group sizes were as follows: control $(n=8), \operatorname{mTBI}(n=6), \operatorname{KD}(n=6)$, and $\mathrm{mTBI}+\mathrm{KD}(\mathrm{n}=5)$.

Elevated plus maze. The elevated plus maze (EPM) was used to evaluate anxiety-like behavior ${ }^{61}$. This assessment relies on the natural anxiety-like behavior exhibited by rodents when placed in brightly lit, open environments. The maze is plus-shaped (+), with arms extending from the center at $90^{\circ}$ angles from each other. Opposite arms in the plus formation are identical, with two open arms and two closed (walled) arms measuring $30 \times 5 \times 1 \mathrm{~cm}$ each. During testing, each mouse was placed at the maze's center, facing one of the open arms, and was allowed to explore the maze for $5 \mathrm{~min}$. The amount of time the mice spent in the open arms and the number of entries to the closed and open arms was counted. A longer duration of time spent within the open arms has been associated with lower anxiety levels ${ }^{61,62}$. Group sizes were as follows: control $(n=19), \operatorname{mTBI}(n=18)$, KD $(\mathrm{n}=23)$, and $\mathrm{mTBI}+\mathrm{KD}(\mathrm{n}=24)$.

Novel object recognition. The novel object recognition (NOR) task assesses recognition and visual memory ${ }^{63}$. This paradigm relies on rodents' natural tendency to investigate novel objects within their environment rather than known ones. NOR evaluates whether a mouse is able to discriminate between a familiar and a novel object. The testing arena is a square surface $(60 \mathrm{~cm} \times 60 \mathrm{~cm})$ with high walls $(20 \mathrm{~cm})$. The test consists of three $5 \mathrm{~min}$ sessions, separated by $24 \mathrm{~h}$. On the first day, mice were individually put in the empty arena for habituation for $5 \mathrm{~min}$. On the second day, the mice were exposed to 2 identical objects within the arena for $5 \mathrm{~min}$. On the third (experimental) day, one of the familiar objects was replaced with a novel object, and mice were allowed to explore the arena again for $5 \mathrm{~min}$, during which time spent near familiar and novel object was measured. The arena was cleaned with $70 \%$ ethanol between subjects. An Aggelton index was calculated as follows: (time near new novel object - time near familiar object)/(time near new novel object + time near familiar object $)^{64}$. A higher Aggelton index indicates advancement in recognition memory. Animals near the objects less than $10 \%$ of the total test time (i.e., less than $30 \mathrm{~s}$ next to the two objects together) were excluded from statistical calculations, as exploration under this amount of time does not allow estimation of subjects' visual memory. Group sizes were as follows: control $(\mathrm{n}=19), \operatorname{mTBI}(\mathrm{n}=18), \mathrm{KD}(\mathrm{n}=23)$, and $\mathrm{mTBI}+\mathrm{KD}(\mathrm{n}=24)$. 


\begin{tabular}{|l|l|l|l|l|l|}
\hline Target/fluorochrome & Primary/secondary & Probe & Manufacturer & Catalog & Dilution \\
\hline Nuclei & & Hoechst 33342 & ThermoFisher & & \\
\hline Astrocytes & Primary & Chicken anti-GFAP & Encor & CPCA-GFA & $1: 1500$ \\
\hline Alexa488 & Secondary & Donkey anti-chicken & Jackson & $703-545-155$ & $1: 500$ \\
\hline Microglia & Primary & Goat anti-Iba1 & ThermoFisher & PA5-18039 & $1: 1500$ \\
\hline Alexa555 & Secondary & Donkey anti-goat & ThermoFisher & A21432 & $1: 500$ \\
\hline NeuN+Neurons & Primary & Rabbit anti-NeuN & Abcam & Ab104225 & $1: 5000$ \\
\hline Alexa647 & Secondary & Donkey anti-rabbit & ThermoFisher & A31573 & $1: 500$ \\
\hline
\end{tabular}

Table 2. Immunohistochemistry reagents.

Y-maze. The Y-maze paradigm was used to evaluate spontaneous exploration, responsiveness to novel environments, and spatial memory function, as previously described ${ }^{65}$. This test relies on the preference of rodents to explore new environments rather than familiar ones. The Y-maze consists of a three-armed black plexiglass maze with arms separated by $120^{\circ}$. Each arm was identical $(8 \times 30 \times 15 \mathrm{~cm})$; however, different spatial cues were placed in each arm (i.e., a triangle, a square, or a circle). In the first session of the test, the mouse was put in the arena's start arm (chosen randomly) and allowed to explore another arm while the third arm was blocked for $5 \mathrm{~min}$. The mouse was then returned to its home cage for $2 \mathrm{~min}$. In the second session, all arms were open for exploration for $2 \mathrm{~min}$ and time spent in each arm was recorded. The arena was cleaned with $70 \%$ ethanol between subjects. The time the mouse spent in the familiar arm and the new arm was measured. An Aggelton index was calculated as follows: (time in the new arm - time in the familiar arm)/(time in new arm + time in the familiar arm) $)^{64}$. A higher Aggelton index indicates improved spatial memory. Group sizes were as follows: control $(n=20)$, mTBI $(\mathrm{n}=19), \mathrm{KD}(\mathrm{n}=24)$, and $\mathrm{mTBI}+\mathrm{KD}(\mathrm{n}=26)$.

Immunohistochemistry. Immunohistochemistry studies were performed hippocampal (dentate gyrus) and temporal cortex tissue sections obtained from animals euthanized on day 30 post-injury. Mice were anesthetized with ketamine $(100 \mathrm{mg} / \mathrm{kg})$ and xylazine $(10 \mathrm{mg} / \mathrm{kg})$ and underwent transcardial perfusion with $10 \mathrm{ml}$ phosphate-buffered saline (PBS) followed by $20 \mathrm{ml}$ of $4 \%$ paraformaldehyde (PFA) in $0.1 \mathrm{M}$ phosphate buffer, $\mathrm{pH}$ 7.4. Brains were removed, fixed overnight in 4\% PFA, and then placed in 1\% PFA. Neuroscience Associates (Knoxville, TN) oriented the brains into a multiblock, collected $35 \mu \mathrm{m}$ sections sequentially through the brains, and performed the floating section staining and mounting (antibodies detailed in Table 2). Microscopy was performed with a Fluoview 3000 laser scanning confocal microscope (Olympus, Waltham, MA). Target locations were determined on a stitched map with only Hoechst 33342 staining captured. For all analyses, regions of interest were selected on the map depicting only the nuclear staining and blinded with respect to groupings. These regions were then collected by multi area time lapse in sequence with the Fluoview 3000 software without intervention. Images, centered on coronal sections at approximately $-2.9 \mathrm{~mm}$ from Bregma, were collected as $\mathrm{Z}$ stacks and maximum $\mathrm{Z}$ projections with constant illumination (405, 488, 561, and $640 \mathrm{~nm}$ diode lasers) and detection parameters. Automated analysis for morphology, intensities, and numbers of cells was conducted using cellSens (Olympus, Waltham, MA, USA) and Image ${ }^{66,67}$.

Changes in the architecture of astrocytes were determined by batch processing of all images collected with the aid of ImageJ. All of the following steps were iteratively executed for each micrograph in a single macro. Each image was analyzed for the channel containing GFAP staining. Background was subtracted with ImageJ's built-in rolling ball process, automated thresholding was accomplished with the built in RenyiEntropy algorithm, and noise was removed using the ImageJ despeckle routine. The image was then skeletonized with the ImageJ plugin andfeatures that were too small to be relevant were removed by the particle remover plugin. The analyze skeleton plugin generated the process lengths per cell. These routines have previously been shown to identify reactive morphology 66,67 .

Frames included in figures are correct concerning orientation, i.e., dorsal at the top, left, and right. Confocal scanning was rotated $30^{\circ}$ to optimize the framing of regions of interest. Group sizes were as follows: control $(\mathrm{n}=5), \operatorname{mTBI}(\mathrm{n}=5), \mathrm{KD}(\mathrm{n}=5)$, and $\mathrm{mTBI}+\mathrm{KD}(\mathrm{n}=5)$.

Western blotting. To assess the cortical and hippocampal SIRT1 levels, brains were dissected following cervical dislocation at 7 and 30 days post-injury. The cortex and hippocampus were separated and frozen in liquid nitrogen, then stored in $-80^{\circ} \mathrm{C}$. Prior to analysis, brains were homogenized in lysis buffer (Tissue Protein Extraction Reagent, Pierce, Waltham, MA, USA) supplemented with a protease inhibitor cocktail (Halt Protease Inhibitor Cocktail, Sigma Aldrich, St. Louis, MO, USA) using a Teflon pestle homogenizer. Homogenates were centrifuged for $15 \mathrm{~min}$ at $4{ }^{\circ} \mathrm{C} 14,000 \mathrm{r} / \mathrm{min}$, supernatant liquids were separated from the precipitates and stored at $-80^{\circ} \mathrm{C}$. Sample buffer was added to the samples and then stored at $-18{ }^{\circ} \mathrm{C}$. Prior to analysis, samples were heated to $90^{\circ} \mathrm{C}$ for $3 \mathrm{~min}$ and $30 \mu \mathrm{l}$ of each sample was then loaded and run on 4-20\% Mini-Protean TGX gels (Bio-Rad, Hercules, CA, USA) followed by transfer onto nitrocellulose membranes (Bio-Rad, Hercules, CA, USA) by a transfer system (Trans-Blot Turbo, Bio-Rad, Hercules, CA, USA). Afterward, blots were blocked for $1 \mathrm{~h}$ at room temperature, with Tris-buffered saline, containing $0.01 \%$ Tween-20 and 5\% BSA or powdered milk. Membranes were then incubated overnight at $4{ }^{\circ} \mathrm{C}$ with a mouse primary anti-SIRT1 antibody (Abcam, Cambridge, UK, ab10304, 1:500) and washed with TBS. Membranes were then incubated at room temperature for 
$1 \mathrm{~h}$ with goat anti-mouse antibody (Jackson ImmunoResearch Laboratories, Inc., West Grove, PA, 115-035-003, 1:10,000). Bands were then exposed using enhanced chemiluminescence with ECL (Millipore, Billerica, MA, United States) for 1 min by Viber Fusion FX7 imaging system (Viber Lourmat, France). Densitometry analysis of the detected signal was performed using ImageJ software. Uniform loading was verified by stripping and reprobing with a mouse primary $\alpha$-tubulin antibody for $30 \mathrm{~min}$ at room temperature (Santa Cruz Biotechnology, Dallas, TX, sc-53030, 1:10,000), then conjugated goat anti-mouse antibody (Jackson ImmunoResearch Laboratories, Inc., West Grove, PA, 115-035-003, 1:10,000). The ratio of SIRT1 and a-tubulin (TUB) determined the value of each sample. Averages of control values in each membrane were set to 1 , and all other samples were calculated accordingly ${ }^{28}$. Figure 3A,B shows cropped blots, the membrane was cropped immediately after the transfer stage due to the usage of two different antibodies on the same blot membrane, SIRT1 band size was at $110 \mathrm{kDa}$ and $\alpha$-tubulin band size was at $55 \mathrm{kDa}$, enabling the process of cropping and incubating two different antibodies due to the distantness from each other without risk of damaging the membranes pristine state. Fulllength blots/gels are presented in Supplementary Figs. 1-4. Group sizes were as follows: control $(n=7)$, mTBI $(n=6), K D(n=5)$, and $\operatorname{mTBI}+\operatorname{KD}(n=7)$.

Statistical analysis. Statistical analysis was carried out using IBM SPSS version 24.0. All values are presented as the mean \pm standard error of the mean (SEM). Statistical analysis included data imputation in order to maximize power, followed by one-way ANOVA, repeated measures ANOVA, or two-way ANOVA where appropriate. Gabriel and Sidak $(\alpha=0.05)$ tests were used as post hoc tests. Partial eta squared $\left(\eta^{2}\right)$ was calculated to show effect size. Significance was determined as a two-sided $p<0.05$. Descriptive statistics for blood ketones are provided in supplementary files, Table 1. Descriptive statistics for SIRT1 expression, Immunohistochemistry, and behavioral data are provided in supplementary files, Table 2.

Ethical approval. The Sackler Commission on Animal Experimentation approved the animal protocol 01-19-058 according to the Guidelines for Animal Experimentation of the National Institutes of Health (DHEW publication 23-85, revised, 1995).

\section{Data availability}

All data supporting this study and its findings are available within the article or from the corresponding author upon reasonable request.

Received: 21 July 2021; Accepted: 18 November 2021

Published online: 07 December 2021

\section{References}

1. Taylor, C. A., Bell, J. M., Breiding, M. J. \& Xu, L. Traumatic brain injury-related emergency department visits, hospitalizations, and deaths-United States, 2007 and 2013. MMWR. Surveill. Summ. 66, 1-16 (2017).

2. Centers for Disease Control and Prevention (CDC). CDC grand rounds: Reducing severe traumatic brain injury in the United States. Morb. Mortal. Wkly. Rep. 62, 549-552 (2013).

3. Rabinowitz, A. R. \& Levin, H. S. Cognitive sequelae of traumatic brain injury. Psychiatr. Clin. North Am. 37, 1-11 (2014).

4. Rao, V. \& Lyketsos, C. Neuropsychiatric sequelae of traumatic brain injury. Psychosomatics 41, 95-103 (2000).

5. Greve, M. W. \& Zink, B. J. Pathophysiology of traumatic brain injury. Mt. Sinai J. Med. 76, 97-104 (2009).

6. Werner, C. \& Engelhard, K. Pathophysiology of traumatic brain injury. Br. J. Anaesth. 99, 4-9 (2007).

7. De Kruijk, J. R., Twijnstra, A. \& Leffers, P. Diagnostic criteria and differential diagnosis of mild traumatic brain injury. Brain Inj. 15, 99-106 (2001).

8. Tashlykov, V. et al. Minimal traumatic brain injury induce apoptotic cell death in mice. J. Mol. Neurosci. 37, 16-24 (2009).

9. Daneshvar, D. H. et al. Long-term consequences: Effects on normal development profile after concussion. Phys. Med. Rehabil. Clin. N. Am. 22, 683-700 (2011).

10. Li, Y. et al. Liraglutide is neurotrophic and neuroprotective in neuronal cultures and mitigates mild traumatic brain injury in mice. J. Neurochem. 135, 1203-1217 (2015).

11. Wheless, J. W. History of the ketogenic diet. Epilepsia 49(Suppl 8), 3-5 (2008).

12. Scichilone, J. M., Yarraguntla, K., Charalambides, A., Harney, J. P. \& Butler, D. Environmental enrichment mitigates detrimental cognitive effects of ketogenic diet in weanling rats. J. Mol. Neurosci. 60, 1-9 (2016).

13. Phillips, M. C. L., Murtagh, D. K. J., Gilbertson, L. J., Asztely, F. J. S. \& Lynch, C. D. P. Low-fat versus ketogenic diet in Parkinson's disease: A pilot randomized controlled trial. Mov. Disord. 33, 1306-1314 (2018).

14. VanItallie, T. B. Biomarkers, ketone bodies, and the prevention of Alzheimer's disease. Metabolism 64, S51-S57 (2015).

15. Hertz, L., Chen, Y. \& Waagepetersen, H. S. Effects of ketone bodies in Alzheimer's disease in relation to neural hypometabolism, $\beta$-amyloid toxicity, and astrocyte function. J. Neurochem. 134, 7-20 (2015).

16. Youm, Y. H. et al. The ketone metabolite $\beta$-hydroxybutyrate blocks NLRP3 inflammasome-mediated inflammatory disease. Nat. Med. https://doi.org/10.1038/nm.3804 (2015).

17. Guo, M. et al. Ketogenic diet improves brain ischemic tolerance and inhibits NLRP3 inflammasome activation by preventing Drp1-mediated mitochondrial fission and endoplasmic reticulum stress. Front. Mol. Neurosci. 11, 86 (2018).

18. Zhang, F., Wu, H., Jin, Y. \& Zhang, X. Proton magnetic resonance spectroscopy (H1-MRS) study of the ketogenic diet on repetitive mild traumatic brain injury in adolescent rats and its effect on neurodegeneration. World Neurosurg. 120, e1193-e1202 (2018).

19. Jarrett, S. G., Milder, J. B., Liang, L.-P. \& Patel, M. The ketogenic diet increases mitochondrial glutathione levels. J. Neurochem. 106, 1044-1051 (2008).

20. Sullivan, P. G. et al. The ketogenic diet increases mitochondrial uncoupling protein levels and activity. Ann. Neurol. 55, 576-580 (2004).

21. Martin, A. et al. Role of SIRT1 in autoimmune demyelination and neurodegeneration. Immunol. Res. 61, 187-197 (2015).

22. Pasinetti, G. M., Wang, J., Ho, L., Zhao, W. \& Dubner, L. Roles of resveratrol and other grape-derived polyphenols in Alzheimer's disease prevention and treatment. Biochim. Biophys. Acta 1852, 1202-1208 (2015).

23. Sasaki, T. et al. Induction of hypothalamic Sirt1 leads to cessation of feeding via agouti-related peptide. Endocrinology 151, 2556-2566 (2010).

24. Ramadori, G. et al. SIRT1 deacetylase in SF1 neurons protects against metabolic imbalance. Cell Metab. 14, 301-312 (2011). 
25. Cohen, D. E., Supinski, A. M., Bonkowski, M. S., Donmez, G. \& Guarente, L. P. Neuronal SIRT1 regulates endocrine and behavioral responses to calorie restriction. Genes Dev. 23, 2812-2817 (2009).

26. Chang, H.-C. \& Guarente, L. SIRT1 mediates central circadian control in the SCN by a mechanism that decays with aging. Cell 153, 1448-1460 (2013).

27. Ran, M. et al. Calorie restriction attenuates cerebral ischemic injury via increasing SIRT1 synthesis in the rat. Brain Res. 1610, 61-68 (2015).

28. Rubovitch, V. et al. Dietary energy restriction ameliorates cognitive impairment in a mouse model of traumatic brain injury. J. Mol. Neurosci. 67, 613-621 (2019).

29. Bele, M. S., Gajare, K. A. \& Deshmukh, A. A. Caloric restriction mimetic 2-deoxyglucose maintains cytoarchitecture and reduces tau phosphorylation in primary culture of mouse hippocampal pyramidal neurons. In Vitro Cell. Dev. Biol. Anim. 51, 546-555 (2015).

30. Dickstein, D. L. et al. Brain and blood biomarkers of tauopathy and neuronal injury in humans and rats with neurobehavioral syndromes following blast exposure. Mol. Psychiatry https://doi.org/10.1038/s41380-020-0674-z (2020).

31. Almeida, M. F. et al. Distinct and dementia-related synaptopathy in the hippocampus after military blast exposures. Brain Pathol. 31, $20(2021)$.

32. Bough, K. J., Valiyil, R., Han, F. T. \& Eagles, D. A. Seizure resistance is dependent upon age and calorie restriction in rats fed a ketogenic diet. Epilepsy Res. 35, 21-28 (1999).

33. Scheibye-Knudsen, M. et al. A high-fat diet and $\mathrm{NAD}(+)$ activate Sirt1 to rescue premature aging in cockayne syndrome. Cell Metab. 20, 840-855 (2014).

34. Yuan, H. et al. The phytochemical epigallocatechin gallate prolongs the lifespan by improving lipid metabolism, reducing inflammation and oxidative stress in high-fat diet-fed obese rats. Aging Cell 19, e13199 (2020).

35. Gräff, J. et al. A dietary regimen of caloric restriction or pharmacological activation of SIRT1 to delay the onset of neurodegeneration. J. Neurosci. 33, 8951-8960 (2013).

36. Zhu, H. et al. Icariin protects against brain injury by enhancing SIRT1-dependent PGC-1a expression in experimental stroke. Neuropharmacology 59(1-2), 70-76 (2010).

37. Hyder, A. A., Wunderlich, C. A., Puvanachandra, P., Gururaj, G. \& Kobusingye, O. C. The impact of traumatic brain injuries: A global perspective. NeuroRehabilitation 22(5), 341-353 (2007).

38. Tamargo, I. A. et al. Novel GLP-1R/GIPR co-agonist "twincretin" is neuroprotective in cell and rodent models of mild traumatic brain injury. Exp. Neurol. 288, 176-186 (2017).

39. Prins, M. L., Fujima, L. S. \& Hovda, D. A. Age-dependent reduction of cortical contusion volume by ketones after traumatic brain injury. J. Neurosci. Res. 82, 413-420 (2005).

40. Hu, Z.-G. et al. The protective effect of the ketogenic diet on traumatic brain injury-induced cell death in juvenile rats. Brain Inj. 23, 459-465 (2009).

41. Zhou, W. et al. The calorically restricted ketogenic diet, an effective alternative therapy for malignant brain cancer. Nutr. Metab. (Lond.) 4, 5 (2007).

42. Stamatovic, S. M. et al. Decline in Sirtuin-1 expression and activity plays a critical role in blood-brain barrier permeability in aging. Neurobiol. Dis. 126, 105-116 (2019).

43. Moreno, C. L. \& Mobbs, C. V. Epigenetic mechanisms underlying lifespan and age-related effects of dietary restriction and the ketogenic diet. Mol. Cell. Endocrinol. 455, 33-40 (2017).

44. Donmez, G. et al. SIRT1 protects against $\alpha$-synuclein aggregation by activating molecular chaperones. J. Neurosci. 32(1), 124-132 (2012).

45. Qubty, D., Rubovitch, V., Benromano, T., Ovadia, M. \& Pick, C. G. Orally administered cinnamon extract attenuates cognitive and neuronal deficits following traumatic brain injury. J. Mol. Neurosci. 71(1), 178-186 (2021).

46. $\mathrm{Mu}$, J. et al. Ketogenic diet protects myelin and axons in diffuse axonal injury. Nutr. Neurosci. 28, 1-14 (2021).

47. Tweedie, D. et al. Mild traumatic brain injury-induced hippocampal gene expressions: The identification of target cellular processes for drug development. J. Neurosci. Methods 272, 4-18 (2016).

48. Bader, M. et al. Pharmacokinetics and efficacy of PT302, a sustained-release Exenatide formulation, in a murine model of mild traumatic brain injury. Neurobiol. Dis. 124, 439-453 (2019).

49. Ratliff, W. A. et al. Sidestream smoke affects dendritic complexity and astrocytes after model mild closed head traumatic brain injury. Cell. Mol. Neurobiol. https://doi.org/10.1007/s10571-020-01036-5 (2021).

50. Ratliff, W. A., Qubty, D., Delic, V., Pick, C. G. \& Citron, B. A. Repetitive mild traumatic brain injury and transcription factor modulation. J. Neurotrauma 37(17), 1910-1917 (2020).

51. Mcdowell, S., Whyte, J. \& D’Esposito, M. Working memory impairments in traumatic brain injury: Evidence from a dual-task paradigm. Neuropsychologia 35(10), 1341-1353 (1997).

52. Stuss, D. T. et al. Subtle neuropsychological deficits in patients with good recovery after closed head injury. Neurosurgery 17(1), 41-47 (1985).

53. Zohar, O. et al. Closed-head minimal traumatic brain injury produces long-term cognitive deficits in mice. Neuroscience 118, 949-955 (2003).

54. Baratz, R. et al. Tumor necrosis factor-a synthesis inhibitor, 3,6'-dithiothalidomide, reverses behavioral impairments induced by minimal traumatic brain injury in mice. J. Neurochem. 118, 1032-1042 (2011).

55. Pan, W., Kastin, A. J., Rigai, T., McLay, R. \& Pick, C. G. Increased hippocampal uptake of tumor necrosis factor alpha and behavioral changes in mice. Exp. brain Res. 149, 195-199 (2003).

56. Baratz, R. et al. Transiently lowering tumor necrosis factor- $\alpha$ synthesis ameliorates neuronal cell loss and cognitive impairments induced by minimal traumatic brain injury in mice. J. Neuroinflamm. 12, 45 (2015).

57. Helenius, T. O. et al. Keratin 8 absence down-regulates colonocyte HMGCS2 and modulates colonic ketogenesis and energy metabolism. Mol. Biol. Cell 26, 2298-2310 (2015).

58. De Feyter, H. M. et al. A ketogenic diet increases transport and oxidation of ketone bodies in RG2 and 9L gliomas without affecting tumor growth. Neuro. Oncol. 18, 1079-1087 (2016).

59. Dhandapani, P. K. et al. Phenotypic effects of dietary stress in combination with a respiratory chain bypass in mice. Physiol. Rep. 7, 14159 (2019).

60. Griffith, C. M., Macklin, L. N., bartke, A. \& patrylo, pr. differential fasting plasma glucose and ketone body levels in GHRKO versus 3xTg-AD mice: A potential contributor to aging-related cognitive status?. Int. J. Endocrinol. 2017, 9684061 (2017).

61. Rodgers, R. J. \& Dalvi, A. Anxiety, defence and the elevated plus-maze. Neurosci. Biobehav. Rev. 21, 801-810 (1997).

62. Fernández Espejo, E. Structure of the mouse behaviour on the elevated plus-maze test of anxiety. Behav. Brain Res. 86, 105-112 (1997).

63. Bevins, R. A. \& Besheer, J. Object recognition in rats and mice: A one-trial non-matching-to-sample learning task to study 'recognition memory'. Nat. Protoc. 1, 1306-1311 (2006).

64. Dix, S. L. \& Aggleton, J. P. Extending the spontaneous preference test of recognition: Evidence of object-location and object-context recognition. Behav. Brain Res. 99, 191-200 (1999).

65. Baratz, R., Rubovitch, V., Frenk, H. \& Pick, C. G. The influence of alcohol on behavioral recovery after mTBI in mice. J. Neurotrauma 27, 555-563 (2010). 
66. Morrison, H. W. \& Filosa, J. A. A quantitative spatiotemporal analysis of microglia morphology during ischemic stroke and reperfusion. J. Neuroinflamm. https://doi.org/10.1186/1742-2094-10-4 (2013).

67. Morrison, H. W. \& Filosa, J. A. Sex differences in astrocyte and microglia responses immediately following middle cerebral artery occlusion in adult mice. Neuroscience 339, 85-99 (2016).

\section{Acknowledgements}

This research was supported in part by the Ari and Regine Aprijaskis Fund at Tel-Aviv University and the Dr. Miriam and Sheldon G. Adelson Chair for the Biology of Addictive Diseases in Tel-Aviv University, Tel-Aviv, Israel. The contents do not represent the views of the Department of Veterans Affairs or the United States Government and the opinions, interpretations, conclusions and recommendations are those of the authors and are not necessarily endorsed by the Department of Defense.

\section{Author contributions}

M.H.-E., V.R. and C.G.P. designed the experiment. M.H.-E. and B.A.C. performed the experiments and analysed the data. V.R. and W.A.R. gave technical support and conceptual advice. B.A.C. supervised the analysis. M.H.-E. wrote the manuscript. W.A.R., V.R. and C.G.P. edited the manuscript. B.R.-H. contributed to analysis of the data. All authors read and approved the final manuscript.

\section{Funding}

This research was funded by the Ari and Regine Aprijaskis Fund, Grant number 347300-00; the Dr. Miriam and Sheldon G. Adelson Center for the Biology of Addictive Diseases, Grant number 601133461; and the Sylvan Adams Sports Institute Grant number 0601133671.

\section{Competing interests}

The authors declare no competing interests.

\section{Additional information}

Supplementary Information The online version contains supplementary material available at https://doi.org/ 10.1038/s41598-021-02849-0.

Correspondence and requests for materials should be addressed to M.H.-E. or C.G.P.

Reprints and permissions information is available at www.nature.com/reprints.

Publisher's note Springer Nature remains neutral with regard to jurisdictional claims in published maps and institutional affiliations.

(c) Open Access This article is licensed under a Creative Commons Attribution 4.0 International License, which permits use, sharing, adaptation, distribution and reproduction in any medium or format, as long as you give appropriate credit to the original author(s) and the source, provide a link to the Creative Commons licence, and indicate if changes were made. The images or other third party material in this article are included in the article's Creative Commons licence, unless indicated otherwise in a credit line to the material. If material is not included in the article's Creative Commons licence and your intended use is not permitted by statutory regulation or exceeds the permitted use, you will need to obtain permission directly from the copyright holder. To view a copy of this licence, visit http://creativecommons.org/licenses/by/4.0/.

(c) The Author(s) 2021 